\title{
Spectra of Local and Nonlocal Two-dimensional Turbulence
}

\author{
RAYMOND T, PIERREHUMBERT \\ Department of Geophysical Sciences, University of Chicago, Chicago, IL 60637, USA
}

and

ISAAC M. HELD

Geophysical Fluid Dynamics Laboratory/NOAA, Princeton University, Princeton, NJ 08540, USA

and

KYLE L. SWANSON

Department of Geophysical Sciences, University of Chicago, Chicago, IL 60637, USA

\begin{abstract}
We propose a family of two-dimensional incompressible fluid models indexed by a parameter $\alpha \varepsilon[0, \infty]$, and discuss the spectral scaling properties for homogeneous, isotropic turbulence in these models. The family includes two physically realizable members. It is shown that the enstrophy cascade is spectrally local for $\alpha<2$, but becomes dominated by nonlocal interactions for $\alpha>2$. Numerical simulations indicate that the spectral slopes are systematically steeper than those predicted by the local scaling argument.
\end{abstract}

Incompressible fluid turbulence in two dimensions is of interest because of its applications in geophysical problems [1] and because of its role as a computationally tractable testbed for theories. Spectrally local similarity theories of the inertial range spectrum based on Kolmogorov's phenomenology [2,3] often provide a starting point for such studies. The enstrophy cascading inertial range in $2 \mathrm{D}$ turbulence has a pathology in this regard; assuming the local similarity spectrum, the aggregate straining effects of eddies larger than a given scale $L$ just fail to be dominated by the effects of eddies with scales similar to $L$. Hence, the enstrophy range of conventional $2 \mathrm{D}$ turbulence is precisely on the threshold of locality $[3,4]$. In this note we examine a family of modified 2D fluid equations, which span the range between spectrally local and strongly nonlocal behavior.

The equation for advection of a conserved scalar $q$ in a velocity field with streamfunction $\psi$ is:

$$
\partial_{,} q+J(\psi, q)=0
$$

where $J(A, B)=\partial_{x} A \partial_{y} B-\partial_{x} B \partial_{y} A$. Advection of even a passive scalar decoupled from $\psi$ embraces a rich variety of phenomena $[5,6]$, but the $2 \mathrm{D}$ hydrodynamic equations are distinguished by coupling between $q$ and $\psi$, which renders the equation nonlinear. The form of the coupling determines the degree of locality. We consider the family of couplings defined in Fourier transform space by 


$$
\widehat{\psi}(\mathbf{k})=-|\mathbf{k}|^{-\alpha} \hat{q}(\mathbf{k}),
$$

where $\mathbf{k}$ is the two-dimensional wavenumber vector, with magnitude $k=|\mathbf{k}|$. Essentially, $\psi(x, y)$ is a smoothed form of $q(x, y)$; as $\alpha$ is made larger, the smoothing becomes more extreme, the advecting field becomes more decoupled from the small-scale structure of the advected field, and the problem becomes more spectrally nonlocal. For $\alpha=2, q$ is vorticity and the equations are the conventional 2D Euler equations. For $\alpha=1$, the equations are also physically realizable; they govern the evolution of three-dimensional stratified rapidly rotating flow with zero potential vorticity in a semi-infinite domain, in which case $q(x, y)$ is identified with the temperature field on the (flat) lower boundary [7]. In terms of the geostrophic streamfunction $\psi(x, y, z, t)$, the boundary temperature is $\partial \psi / \partial z$ at $z=0$. $\psi$ satisfies a three-dimensional Laplace equation, and the problem is closed by imposing decay conditions at $z \rightarrow \infty$. This leads to the spectral space expression (1b) with $\alpha=1$.

Equations ( $1 \mathrm{a}, \mathrm{b})$ have two quadratic conserved quantities, namely, mean energy and enstrophy, defined, respectively, by:

$$
\bar{E}=-\frac{1}{A} \iint \psi q \mathrm{~d} x \mathrm{~d} y \quad \text { and } \quad \bar{G}=\frac{1}{A} \iint q^{2} \mathrm{~d} x \mathrm{~d} y
$$

in which $A$ is the area of the domain. The power spectra $E(k)$ and $G(k)$ are defined so that

$$
\bar{E}=\int_{0}^{\infty} E \mathrm{~d} k \quad \text { and } \quad \bar{G}=\int_{0}^{\infty} G \mathrm{~d} k
$$

with $G=k^{\alpha} E=k^{2(\alpha-1)} V$, where $V$ is the power spectrum of the velocity field. If $\alpha=2$, $E=V ;$ if $\alpha=1, G=V$. The spectral fluxes are defined by $\partial_{t} E=-\partial_{k} F_{\mathrm{E}}$ and $\partial_{t} G=$ $-\partial_{k} F_{G}$. In equilibrium, both $F_{E}$ and $F_{G}$ must be constant.

The dimensions of $F_{E} / F_{G}$ are $L^{2 \alpha}$. The fundamental tenet of the Kolmogorov-Kraichnan scale analysis is that the only available length scale is the local eddy scale $k^{-1}$. Hence, since the fluxes must be independant of $k$, only one of $F_{E}$ and $F_{G}$ can be nonzero. Nonzero $F_{E}$ yields the energy cascading spectrum; since the dimensions of $E$ are $L^{5-\alpha} T^{-2}$ and the dimensions of $F_{E}$ are $L^{4-a} T^{-3}$, dimensional analysis implies

$$
E=a(\alpha)\left(F_{E}\right)^{2 / 3} k^{\gamma-a}, \quad G=a(\alpha)\left(F_{E}\right)^{2 / 3} k^{\gamma}
$$

with $\gamma=-\frac{7}{3}+\frac{4}{3} \alpha$. This yields the conventional $k^{-5 / 3}$ energy spectrum for $\alpha=2$. Following similar reasoning, the spectrum in the enstrophy cascading range is

$$
E=a(\alpha)\left(F_{G}\right)^{2 / 3} k^{\gamma-\alpha}, \quad G=a(\alpha)\left(F_{G}\right)^{2 / 3} k^{\gamma}
$$

with $\gamma=-\frac{7}{3}+\frac{2}{3} \alpha$. For $\alpha=2$, this yields the customary $k^{-1}$ enstrophy spectrum, while for $\alpha=1$, the enstrophy spectrum is $k^{-5 / 3}$. The enstrophy spectrum is integrable at shortwaves whenever $\alpha<2$, but diverges like $k^{(2 / 3) \alpha}$ for large $\alpha$. The spectral shape for $\alpha=1$ was first obtained ref. [7], and has been reproduced in EDQNM closure model simulations [8].

Following Kraichnan $[3,4,5,9]$ the strain rate due to eddies with scales between $1 / k$ and $2 / k$ is $k V(k V)=k^{2-\alpha} V(k G)$. Substituting (5) for the spectrum, we find that the enstrophy cascade is dominated by local strain for $\alpha<2$ and dominated by large eddy strain for $\alpha>2$. Notably, the $k^{-1}$ local scaling spectrum for $\alpha=2$ is the same as the passive scalar spectrum predicted $[6,10]$ for the strongly nonlocal case in which straining is dominated by large eddies with a fixed timescale. Thus, the spectral behavior shades continuously over to the nonlocal scaling as $\alpha$ approaches 2 from below. This is because the conserved quantity $q$ is coincidentally the straining rate for $\alpha=2$. Strong deviations from the predictions of local scaling are expected for $\alpha>2$.

Making use of (4), the strain-rate scaling for the energy cascading range is found to be 
$k^{4 / 3-\alpha / 3}$, whence, as is well known, the cascade is already local for $\alpha=2$. The energy cascading range does not become spectrally nonlocal until $\alpha$ exceeds 4 .

Advection of a tracer by a time-varying large-scale flow typically produces filaments whose thickness decreases exponentially with time $[5,6]$. The stability properties of such filaments shed further light on the locality of the enstrophy cascade. Consider a filament with profile $q(\mathrm{y})=q_{\max } f(y / L)$, which is to be thought of as a local representation of a curved filament which is varying slowly in time compared to the growth of instabilities upon it. If $(1 a, b)$ is linearized about this profile, it follows from dimensional analysis that the wavelength of maximum instability scales with $L$, and that the maximum growth rate of instabilities scales with $q_{\max } L^{\alpha-2}$. Large-scale straining with rate $\varepsilon$ stabilizes instabilities with growth rates less than $O(\varepsilon)$ (see the discussion in ref. [11] for $\alpha=2$ ). Thus, in the presence of large eddy-strain of a given magnitude, filaments always succumb to a secondary instability for $\alpha<2$, instantiating the classical 'large swirls have smaller swirls' cascade. For $\alpha>2$ the large-scale strain always suppresses the instability. In the marginal case $\alpha=2$, whether or not the filament undergoes secondary rollup depends on the initial condition, via $q_{\max } / \varepsilon$.

The scaling of the instability suggests the possibility of finite-time singularities for $\alpha<2$. Filament thickness decreases exponentially in time at the local strain-rate, but the local strain is roughly proportional to the growth rate of the filament instability (which creates the strain). Substituting the instability scaling yields $\mathrm{d} L / \mathrm{d} t=-a q_{\max } L^{\alpha-1}$. Thus, a filament with initial width $L_{0}$ collapses to zero thickness at time $\left(L_{0}^{2-\alpha}\right) /\left((2-\alpha) a q_{\max }\right)$. This agrees with the result derived in ref. [8] for $\alpha=1$. As $\alpha \rightarrow 2^{-}$, the blowup time diverges and we recover global regularity.

We carried out a series of numerical simulations of $1(\mathrm{a}, \mathrm{b})$ with $\alpha=0.5,1,2$ and 3 . The simulations were carried out using a de-aliased spectral model with $512^{2}$ resolution and $\nabla^{8}$ dissipation. It was forced by holding the vorticity amplitudes for modes with wavenumbers between 6 and 8 fixed, with random, time-dependent phases. The enstrophy spectra are shown in Fig. 1, and the spectral slopes calculated in the wavenumber range $10<k<100$ are summarized in Table 1. These do not represent true equilibrium results, as the inverse energy cascade has not proceeded to completion; the slopes in the enstrophy cascading range have nonetheless converged. A calculation carried out at $256^{2}$ resolution revealed essentially the same inertial range slopes.

As predicted by local similarity theory, the spectra become steeper as $\alpha$ is decreased. However, the simulated spectra are systematically steeper than the similarity prediction. The difficulties in obtaining the local similarity spectrum for conventional $2 \mathrm{D}$ turbulence

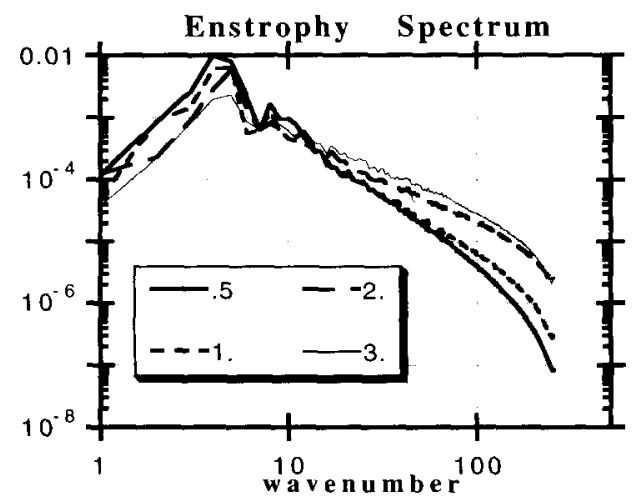

Fig. 1. Power spectra for $\alpha=0.5,1,2$ and 3 . 


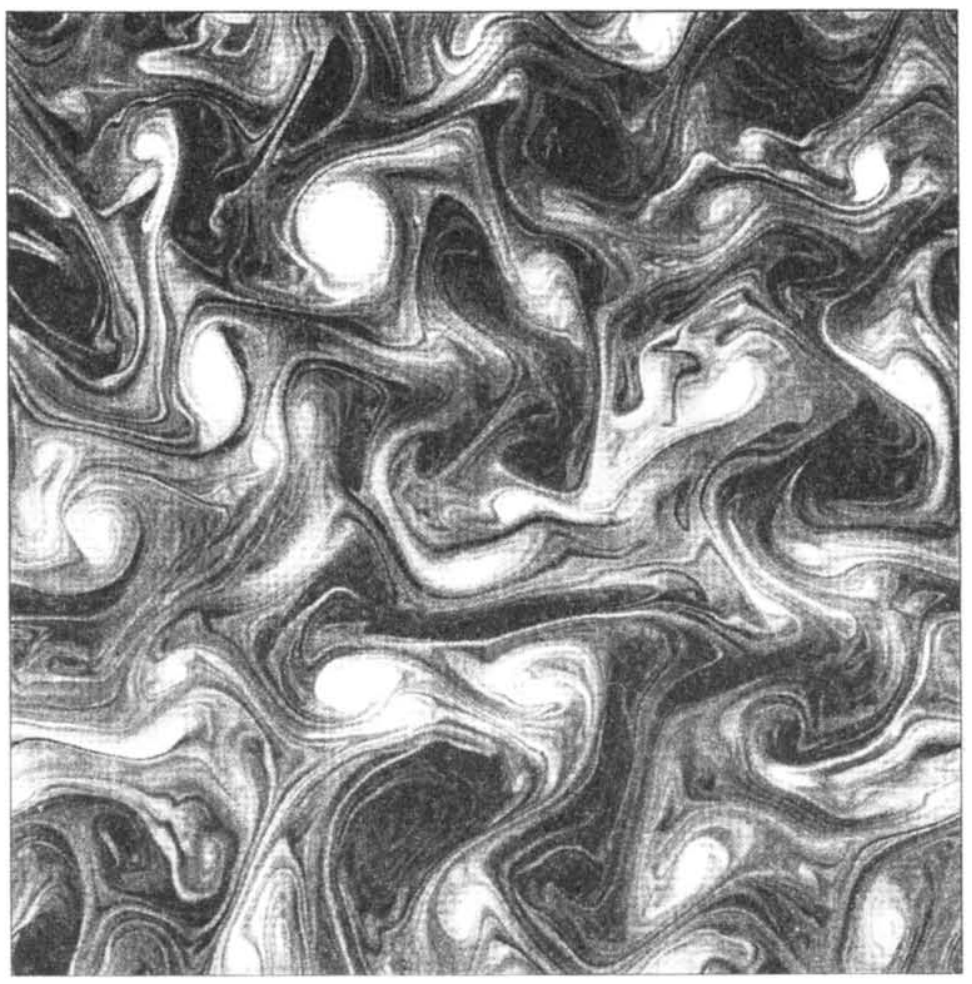

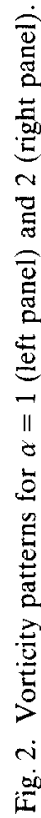

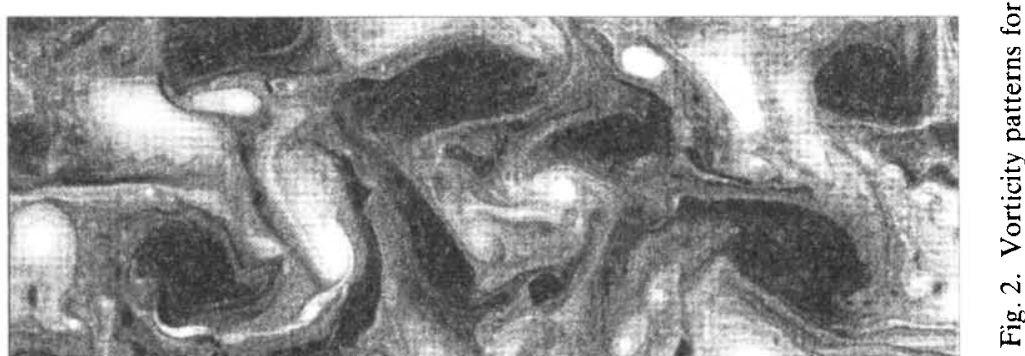


Table 1.

\begin{tabular}{lcc}
\hline$\alpha$ & Slope (actual) & Slope (theory) \\
\hline 0.5 & -2.2 & -2.0 \\
1 & -1.9 & -1.7 \\
2 & -1.4 & -1.0 \\
3 & -1.3 & -0.33 \\
\hline
\end{tabular}

have been discussed extensively in the literature (e.g. ref. [12]). The generalized case we have studied exhibits the same anomaly, despite the unambiguous spectral locality for $\alpha<2$. The EDQNM model [8] does not yield an anomalously steep spectrum for $\alpha=1$; this may reflect a shortcoming in the closure scheme, or it may be indicative of insufficient resolution of the inertial range in our calculation. Intermittency corrections [13], or coherent vortex formation [14] may also come into play in steepening the spectra. As $\alpha$ is increased beyond 2, the local similarity theory predicts that the slope continues to become more shallow. In reality, the slope for $\alpha=3$ is nearly the same as that for $\alpha=2$, suggesting a dominance of the enstrophy cascade by spectrally nonlocal large eddy advection, and a crossover to Batchelor-type passive scalar behavior.

Images of the vorticity fields for $\alpha=1$ and 2 are shown in Fig. 2, and show the decreasing importance of secondary instability and small vortex formation as $\alpha$ increases. For $\alpha=1$, one sees a mix of small vortices, 'curdled' filaments and undisturbed filaments. The vortices arising from instability of the filaments are nearly two orders of magnitude smaller than the primary vortices at the injection range. This suggests that the finite-time collapse discussed above emerges from a sequence of large, discrete spectral jumps, of which our calculation has resolved only the first. For $\alpha=2$ the scene is dominated by large vortices, large spirals, and smooth filaments. Given the marginal locality in this case, the ability of the filaments to resist strain is probably dependent on the details of the forcing. An example showing the coexistence of passively strained filaments with filaments exhibiting strong rollup in conventional $2 \mathrm{D}$ turbulence is given in ref. [15].

It appears that the anomalous spectral steepness commonly found in two-dimensional turbulence is not closely related to spectral nonlocality. On the one hand, $\alpha=2$ behaves as if the cascade were dominated by large-scale advection, for which Batchelor's argument gives a $k^{-1}$ enstrophy spectrum. Thus, Batchelor's phenomenology is failing; the reasons for the failure can be most clearly studied in the context of large eddy passive advection problems such as Ottino [5] or Pierrehumbert [6]. On the other hand, the $\alpha<2$ cases exhibit anomalous steepness despite spectral locality; the reasons for this breakdown can be most clearly studied in the $\alpha=1$ model, for which the issue is disentangled from the marginal locality bedeviling $\alpha=2$.

\section{REFERENCES}

1. P. B. Rhines, Geostrophic turbulence, Ann. Rev. Fluid Mech. 11, 401-442 (1979).

2. A. N. Kolmogorov, The local structure of turbulence in incompressible fluid at very high Reynolds number, Dokl. Akad. Sci. USSR, 30, 299-303 (1941).

3. R. Kraichnan, Eddy viscosity in two and three dimensions, J. Atmos. Sci. 33, 1521-1536 (1976).

4. R. Kraichnan, Inertial-range transfer in two- and three-dimensional turbulence, J. Fluid Mech. 47, 525-535 (1971).

5. J. Ottino, The Kinematics of Mixing: Stretching, Chaos and Transport. Cambridge University Press, Cambridge (1989).

6. R. T. Pierrehumbert, Chaotic mixing of tracer and vorticity by modulated travelling Rossby waves, Geophys Astrophys. Fluid Dynamics 58, 285-319 (1991).

7. W. Blumen, Uniform potential vorticity flow-Part I: Theory of wave interactions and two-dimensional turbulence, J. Atmos. Sci. 35, 774-783 (1978). 
8. J.-M. Hoyer and R. Sadourny, J. Atmos. Sci. 39, 707-721 (1982).

9. R. Kraichnan, Inertial ranges in two-dimensional turbulence, Phys, Fluids 10, 1417-1423 (1967).

10. G. K. Batchelor, Small-scale variation of convected quantities like temperature in turbulent fluid-Part 1; General discussion and the case of small conductivity, J. Fluid Mech. 5, 113-133 (1959).

11. D. G. Dritschel, P. H. Haynes, M. N. Juckes and T. G. Shepherd, The stability of a two-dimensional vorticity filament under uniform strain, J. Fluid Mech. 230, 647-665 (1991).

12. M. E. Brachet, M. Meneguzzi and P. L. Sulem, Small-scale dynamics of high Reynolds number twodimensional turbulence, Phys. Rev. Lett. 57, 683-686 (1986).

13. C. Basdevant, B. Legras, R. Sadourny and M. Beland, A study of barotropic model flows: Intermittency, waves and predictability, J. Atmos. Sci. 38, 2305-2326 (1981).

14. J. Herring and R. Kraichnan, J. Fluid Mech. 153, 229-242 (1985).

15. M. N. Juckes and M. E. McIntyre, A high resolution, one-layer model of breaking planetary waves in the stratosphere, Nature Lond. 328, 590-596 (1987). 